\title{
Concepts for a high resolution multi-object spectrograph for galactic archeology on the Anglo-Australian Telescope
}

\author{
Samuel C. Barden ${ }^{\mathrm{a}}$, Joss Bland-Hawthorn ${ }^{\mathrm{b}}$, Vladimir Churilov ${ }^{\mathrm{a}}$, Simon Ellis ${ }^{\mathrm{a}}$, Tony Farrell ${ }^{\mathrm{a}}$, Ken C. \\ Freeman $^{\mathrm{c}}$, Roger Haynes ${ }^{\mathrm{a}}$, Anthony Horton ${ }^{\mathrm{a}}$, Damien J. Jones ${ }^{\mathrm{d}}$, Greg Knight ${ }^{\mathrm{e}}$, Stan Miziarski ${ }^{\mathrm{a}}$, \\ William Rambold ${ }^{\mathrm{a}}$, Greg Smith ${ }^{\mathrm{a}}$, Lew Waller ${ }^{\mathrm{a}}$ \\ ${ }^{a}$ Anglo-Australian Observatory, PO Box 296, Epping NSW 1710, Australia; \\ ${ }^{\mathrm{b}}$ School of Physics, The University of Sydney NSW 2006, Australia; \\ ${ }^{c}$ Research School of Astronomy \& Astrophysics, Mount Stromlo Observatory, Cotter Road, Weston \\ ACT 2611, Australia; \\ 'Prime Optics, 17 Crescent Road, Eumundi, QLD 4562, Australia; \\ eSinclair Knight Merz, 100 Christie Street, Sydney NSW 2065, Australia
}

\begin{abstract}
Mapping out stellar families to trace the evolutionary star formation history of the Milky Way requires a spectroscopic facility able to deliver high spectral resolution ( $\mathrm{R} \geq 30 \mathrm{k})$ with both good wavelength coverage ( 400 Ang) and target multiplex advantage ( $\sim 400$ per 2 degree field). Such a facility can survey 1,200,000 bright stars over 10,000 square degrees in about 400 nights with a 4-meter aperture telescope. Presented are the results of a conceptual design study for such a spectrograph, which is under development as the next major instrument for the Anglo-Australian Observatory. The current design (that builds upon the AAOmega system) makes use of a White Pupil collimator and an R3 echelle that is matched to the existing AAOmega cameras. The fibre slit can be reconfigured to illuminate the Pupil relay side of the collimator mirror bypassing the echelle, thus preserving the lower dispersion modes of the AAOmega spectrograph. Other spectrograph options initially considered include use of an anamorphic collimator that reduces the required dispersion to that achievable with VPH grating technology or possible use of a double-pass VPH grating.
\end{abstract}

Keywords: spectrograph, high resolution, echelle, multi-object, white pupil

\section{INTRODUCTION}

The High Resolution Multi-Object Echelle Spectrograph (HERMES) is an instrument for a massive survey of 1.2 million stars in the Milky Way Galaxy over 10,000 square degrees on the sky to measure chemical abundances and kinematics. Such a survey will reveal families of stars that formed together, but were then dispersed by the gravitational well of the Galaxy. Tracing the kinematic behavior of each stellar family will yield insight into the formation processes that created the present day structure of the Milky Way ${ }^{[1],[2],[3]}$.

The fundamental requirements for such an instrument are:

- $\quad$ spectral resolving power of $\geq 30,000$

- $\quad$ simultaneous wavelength coverage of 400 Ångstroms within 5000-9000 Ångstrom spectral window

- $\quad$ 400 simultaneous targets over 2 degree field of view

The Australian astronomical community selected HERMES as the most desirable new instrument for the AngloAustralian Telescope in the coming decade at a community workshop held in late 2007. Major funding for the instrument was secured by the Australian government through the National Collaborative Research Infrastructure Strategy (NCRIS) scheme. The instrument recently passed a conceptual design review in May 2008 and is now entering a preliminary design phase. It is projected to be ready for commissioning in late 2011/early 2012.

Ground-based and Airborne Instrumentation for Astronomy II, edited by lan S. McLean, Mark M. Casali, Proc. of SPIE Vol. 7014, 70144J, (2008) · 0277-786X/08/\$18 · doi: 10.1117/12.788549 


\section{SCIENCE REQUIREMENTS}

In addition to the science objectives that initially defined the instrument, HERMES is also expected to serve as a general purpose, facility class instrument. A science workshop on HERMES concluded that there should be an additional mode in which the resolving power was higher, but that the target density is lower allowing the possible use of cross-dispersion to increase the wavelength coverage. Table 1 details the initial science requirements on these main observing modes for HERMES.

Table 1. Requirements.

\begin{tabular}{|c|c|c|c|}
\hline Item & Mode & Requirement & Comment \\
\hline Field of View & Both & 2 degrees & $\begin{array}{l}\text { FoV and simultaneous targets } \\
\text { per exposure are good match to }\end{array}$ \\
\hline \multirow[t]{2}{*}{ Number of Objects } & High Res & $\sim 400$ & existing $2 \mathrm{dF}$ facility. \\
\hline & Very High Res & $\sim 40$ & \\
\hline \multirow[t]{2}{*}{ Spectral Resolving Power } & High Res & 30,000 nominal & $\lambda / \Delta \lambda \mathrm{FWHM}$ \\
\hline & Very High Res & 40,000 to 50,000 & \\
\hline \multirow[t]{2}{*}{ Simultaneous $\lambda$ Coverage } & High Res & $\sim 400 \AA$ & \multirow{2}{*}{$\begin{array}{l}\text { Preferably in two comparable } \\
\text { swaths }\end{array}$} \\
\hline & Very High Res & $\sim 2000 \AA$ & \\
\hline Operable $\lambda$ Range & Both & $3900-9500 \AA$ & Non-simultaneous \\
\hline Sensitivity & Both & $\begin{array}{l}\mathrm{SNR}=100 \text { in } 60 \text { minutes } \\
\text { at } \mathrm{V}=14\end{array}$ & SNR is per resolution element \\
\hline
\end{tabular}

\section{INSTRUMENT DESIGN OPTIONS}

A variety of design options were explored before finalizing on the current design choice.

\subsection{Dual VPH Concept}

One concept considered was whether or not the existing AAOmega spectrograph ${ }^{[4],[],[6]}$ could be retrofitted with a double-pass VPH grating. The high efficiencies offered by VPH gratings and potentially simple solution made this especially worth considering. The idea was that it might be possible to design a special VPH grating with a set of parallel prisms mounted on the back that served as reflectors for the beam so that the light passes through the grating twice and in such a way so as to effectively double the dispersion.

It was realized that the current set of high resolution gratings operated in this mode would provide roughly $\mathrm{R}=22,000$ spectral resolution. Alternatively, the use of immersion prisms was considered to possibly boost this up to nearly 30,000 .

Continued study on this approach was discontinued:

- Alignment tolerances required of the prisms may be too tight and quite difficult to achieve as they would be similar to those required for ultra-precision retroreflectors.

- The prisms need to be phased in wavelength so that the full width of the grating is setting the resolving power rather than just the width of each prism.

- This approach doesn't easily achieve the spectral resolution required for HERMES.

- This approach wouldn't meet the wavelength coverage required for HERMES unless the spectral resolution was allowed to be reduced. This is a matter of only having $\sim 4,000$ total pixels in the two AAOmega cameras and a sampling of over 3 pixels per resolution element. 


\subsection{Classical non-White Pupil Echelle Concept}

Echelle spectrographs dating from the 1960's through the early 1990's were based upon non-Littrow configurations and made use of the anamorphic magnification of the grating to further enhance the spectral resolving power of the instrument. An excellent example of the use of that concept in today's instruments is the design of the Hectochelle spectrograph at the $\mathrm{MMT}^{[8],[9],[10],[11]}$. It is a classical non-Littrow arrangement that provides relative simplicity in the design and minimizes the optical componentry.

However, the following attributes of such a design were identified as concerns for HERMES:

- The camera optics must be large enough to adequately capture the light stretched by the anamorphic factor. This would require custom cameras and would exclude the possible use of existing AAOmega components resulting in likely higher costs for the instrument.

- The wavelength coverage $(\sim 400 \AA)$ would not easily be achieved within a single order of the echelle. Implementing multiple channels into such a design would likely be complex and difficult due to the wavelength separation of the pupil after the light is dispersed by the grating. Likewise, allowing some cross-dispersion while maintaining the $\sim 400$ targets would also be difficult. Space issues would likely become severe with this approach.

\subsection{Anamorphic Collimator Concept}

A recent and interesting development in high resolution spectrograph design is the use of anamorphic optics within the collimator to stretch the beam in the spectral dimension before it illuminates the grating. This then allows one to use a lower angle of diffraction. The CODEX instrument originally envisioned for implementation on the OWL telescope is one example for this design approach for high resolution spectroscopy ${ }^{[12]}$.

The advantage that this approach might have is the ability to utilize VPH gratings operating with a Bragg angle of $\sim 45$ degrees where diffraction efficiencies can be quite high ( $90 \%)$.

Again, although this approach is intriguing and might lead to a very efficient instrument, it has been rejected on the following grounds:

- The collimator would be inappropriate for possible utilization of existing AAOmega components. HERMES would have to be a completely separate instrument.

- If VPH gratings were utilized, the wavelength coverage would likely not be achieved with a single grating. Multiple channels would require multiple gratings.

- The physical length of the grating would be quite large ( $\sim 1$ meter for a $280 \mathrm{~mm}$ initial beam size) and might cause problems with fabrication or cost of the grating.

- Initial exploration also suggested that there may be difficulties getting good performance with large field angles on the slit.

\subsection{White Pupil Design Concept}

A white pupil design ${ }^{[13],[14]}$ was explored and deemed the most viable design choice as it allows the possibility for utilizing and interfacing to existing AAOmega components. It also provides a relatively easy means for switching modes of operation between HERMES high resolution use or pre-existing AAOmega capability. The remainder of this paper will focus on this design option and will address the issues associated with a white pupil implementation.

\section{INSTRUMENT DESIGN}

\subsection{Design Assumptions}

The design presented here assumes that HERMES will utilize the following existing components of the Anglo-Australian Telescope:

- $2 \mathrm{dF}$ corrector, fibre positioner, and fibres

- Interface with AAOmega and occupy the same lab space as AAOmega 
It is currently thought that interfacing HERMES to AAOmega would be the most cost effective approach, however there are implementation issues that may make it desirable to have HERMES built as a completely separate instrument located elsewhere. Usage of AAOmega allows HERMES access to the two existing Schmidt cameras, the existing fibre cable, and the spectrograph lab. If HERMES is to make such use of AAOmega, then an added requirement on HERMES is that it not seriously impact existing AAOmega functionality and performance. In other words, HERMES must have a low resolution mode. On the other hand, if HERMES is a stand alone instrument, then HERMES will require all new hardware and fibre cables.

The existing AAT, 2dF, and AAOmega facilities provide the characteristics listed in Table 2.

Table 2. The AAT, 2dF, and AAOmega parameters (see [4], [5], and [6]).

\begin{tabular}{|lrl|}
\hline Primary Mirror Aperture Diameter & 3900 & $\mathrm{~mm}$ \\
Primary Mirror Curvature Radius & $2.54 \mathrm{E}+04$ & $\mathrm{~mm}$ \\
Prime Focus Focal Ratio & 3.424 & \\
Inverse Plate Scale & 0.06474 & $\mathrm{~mm} / \mathrm{arc}-\mathrm{sec}$ \\
Plate Scale & 15.4464 & $\mathrm{arc}-\mathrm{sec} / \mathrm{mm}$ \\
Prime Focus Field Diameter (physical) & 480.3 & $\mathrm{~mm}$ \\
Prime Focus Field Diameter (on-sky) & 2 & degrees \\
Fibre Diameter (physical) & 140 & microns \\
Fibre Diameter (on-sky) & 2.16 & arc-sec \\
Number of Fibres & 392 & \\
Wavelength Range & $0.37-0.95$ & $\mathrm{~nm}$ \\
Spectrograph Beam Size & 190 & $\mathrm{~mm}$ \\
Number of Channels (Cameras) & 2 & \\
Collimator Type & Schmidt & \\
Collimator Focal Ratio & 3.15 & \\
Camera Types & Schmidt & \\
Camera Focal Ratio & 1.3 & \\
CCD pixel size & 15 & microns \\
CCD format (EEV 44-82) spectral & 2048 & pixels \\
CCD format (EEV 44-82) spatial & 4096 & pixels \\
Nominal Fibre Sampling on Detector & 3.8 & pixels \\
Dispersing Element Type & VPH Gratings & \\
Camera Articulation Range & $0-94$ & degrees \\
\hline
\end{tabular}

\subsection{Modes of Operation}

The HERMES concept has the following three primary operating modes:

1. Low Resolution or Classic AAOmega Mode ( $\mathrm{R}=1300$ to 11000$)$

2. High Resolution Mode $(\mathrm{R} \geq 30000)$

3. Very-High Resolution Mode with cross-dispersion and a limited set of fibres $(\mathrm{R}>40,000)$

Each of these modes requires a reconfiguration of the optics. Reconfiguration between these modes will only occur during the daytime. There may be the possibility of blended modes (e.g. use of the cross-disperser and limited fibres with the High Resolution Mode).

At present, the Very-High Resolution Mode is an optional upgrade, but effort is underway to ensure adequate design to allow future implementation.

\subsection{Design Parameters}

Table 3 lists the parameters for the HERMES concept beyond those already provided in Table 2. 
Table 3. The additional HERMES parameters.

\begin{tabular}{|lrr|}
\hline Number of Channels (Cameras) & 4 & \\
Collimator Type & Houghton derivative & \\
Collimator Focal Ratio & White Pupil & \\
Fiber Relay & 9.0 & \\
Camera Types & $\mathrm{f} / 3.15$ to f/9.0 & \\
& 2 Schmidt & \\
Camera Focal Ratio & 2 Transmissive & \\
CCD pixel size & 1.3 & \\
CCD format (EEV 44-82) spectral & 15 & microns \\
CCD format (EEV 44-82) spatial & 2048 & pixels \\
Nominal Fibre Sampling on Detector & 4096 & pixels \\
Dispersing Element Type & 3.8 & pixels \\
Grating Line Frequency & echelle & \\
Grating Blaze Angle & 110 & l/mm \\
\hline
\end{tabular}

\subsection{High Resolution Mode}

The High Resolution Mode is that mode used to achieve the primary science objectives of the Milky Way stellar survey. The baseline concept utilizes a White Pupil, Houghton derivative design (spherical mirrors and correcting elements) in which the fibre slit is oriented and aligned to illuminate the echelle side of the collimator. A $110 \mathrm{l} / \mathrm{mm} \mathrm{R} 3$ echelle is installed to disperse the light. The collimator accepts the light diffracted by the echelle and reimages it onto an intermediate slit mirror located next to the input fibre slit. That mirror then relays the light to the Pupil relay side of the collimator, which recollimates the light and couples it into the AAOmega cameras.

The AAOmega cameras are positioned and fixed at zero degrees articulation angle. Two additional transmissive cameras are installed, one in each AAOmega channel, to provide a total of four camera channels for this mode of operation. The AAOmega VPH gratings are removed and replaced with upward looking dichroics that split the light in wavelength with one portion of wavelength proceeding to the AAOmega camera while the other portion is directed upwards into one of the new transmissive cameras.

Wavelength separation is achieved through the use of the three beam splitters that separate the spectrum into the four channels plus an additional order separation filter in front of each camera that limits the wavelength bandwidth for each channel. These beam splitters and order filters will be exchangeable to allow access to other wavelength regions.

A custom ruled echelle mosaic $(190 \times 570 \mathrm{~mm})$ is required for this mode. Early exploration of the use of existing echelle formats showed that a $110 \mathrm{l} / \mathrm{mm}$ R2 echelle would suffice. However, it would require a beam diameter of $300 \mathrm{~mm}$ to achieve the full resolution. This causes the White Pupil collimator to be asymmetric in order to decrease to the $190 \mathrm{~mm}$ beam of the existing cameras. With an asymmetric collimator, switching to the Low Resolution Mode becomes more problematic as the slit curvature is different and the Houghton derivative corrector optics must be swapped. The added cost and complexity would far outweigh the costs associated with the production of a custom grating.

Figure 1 shows the optical layout of the concept in the High Resolution Mode of operation.

\subsection{Low Resolution Mode (Classic AAOmega Mode)}

This mode will provide effectively the same functionality as is currently achieved with the AAOmega spectrograph. The two cameras will retain full articulation and all current AAOmega VPH gratings can be utilized. The observer should see little, if any difference in performance or operation of this mode from that currently achieved.

The existing AAOmega Schmidt collimator is replaced with the Houghton derivative White Pupil Collimator as described in the High Resolution Mode. However, in Classic AAOmega mode, the fibres are positioned and aimed such that only the pupil relay side of the collimator is illuminated to collimate the light and feed it into the AAOmega cameras. The echelle grating is not used and is removed in order to allow full articulation of the AAOmega cameras. Likewise, the two transmissive cameras are also removed. The upward looking dichroic beamsplitters are removed and replaced with the desired VPH gratings. Figure 2 shows the schematic for the Low Resolution Mode of operation. 


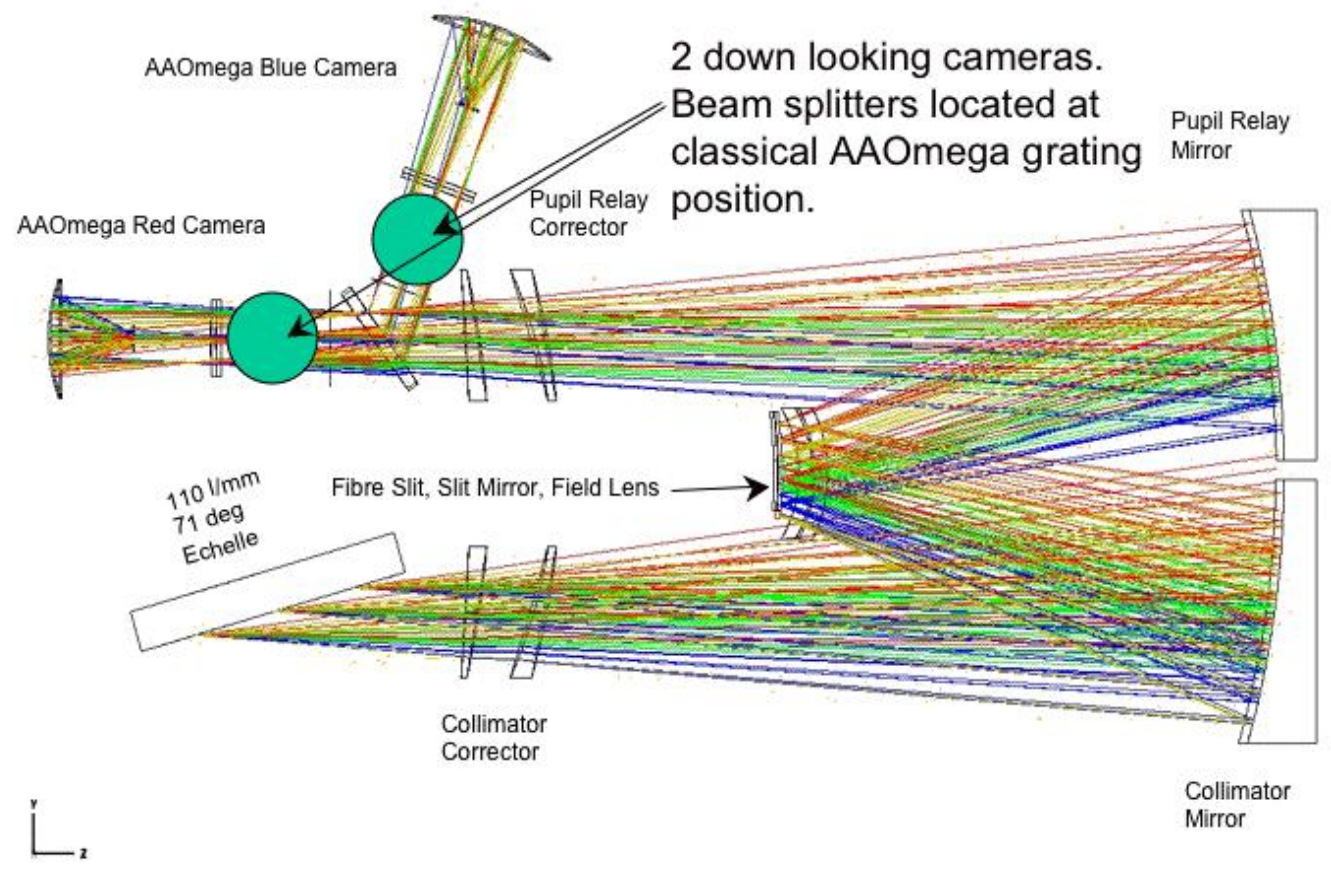

Fig 1. Optical schematic of the HERMES concept in the High Resolution Mode. The light exiting the fibre slit hits the lower half of the collimator mirror. The collimated light passes to the echelle, is dispersed and redirected back to the lower half of the collimator mirror. The mirror forms an initial image of the dispersed spectra on the slit mirror, which reflects the light towards the upper half of the collimator mirror (the pupil relay mirror). The light then enters the modified AAOmega unit and is separated into four channels via beam splitters. The final spectra are imaged onto the four cameras (two Schmidt and two transmissive).

\subsection{Very High Resolution Mode}

In order for HERMES to serve as a facility instrument, it is expected to serve additional scientific applications other than that required for the main stellar survey. These additional science drivers push for higher spectral resolution with higher wavelength coverage, but for a smaller set of targets.

By changing to either an R4 or R5 echelle, adding a cross disperser and masking off 9 out of 10 fibres, HERMES can achieve the 40 fibre Very-High Resolution Mode. This option adds considerable additional cost over the basic instrument with the need for a second echelle mosaic and grating handling equipment. Alternatively, a slit mask could be utilized to narrow down the entrance slit aperture while still using the R3 echelle. This is viable since the High Resolution Mode fibres subtend 3.8 pixels on the detector. Image quality with the existing AAOmega cameras is better than 2 pixels, so a reduced slit width could improve the resolving power of the instrument by a factor of 1.5 to nearly 2 . This same slit mask could also mask out the 9 out of 10 fibres for cross-dispersion. A cross-disperser will still be required for the added spectral coverage. It is likely that only the two AAOmega Schmidt cameras would be used for this mode.

In order to allow a removable cross-disperser, the cross-disperser would need to provide zero deviation of the main spectrograph beam. Further design development will be based upon the zero deviation design previously explored by the Hectochelle project ${ }^{[8],[9]}$. 


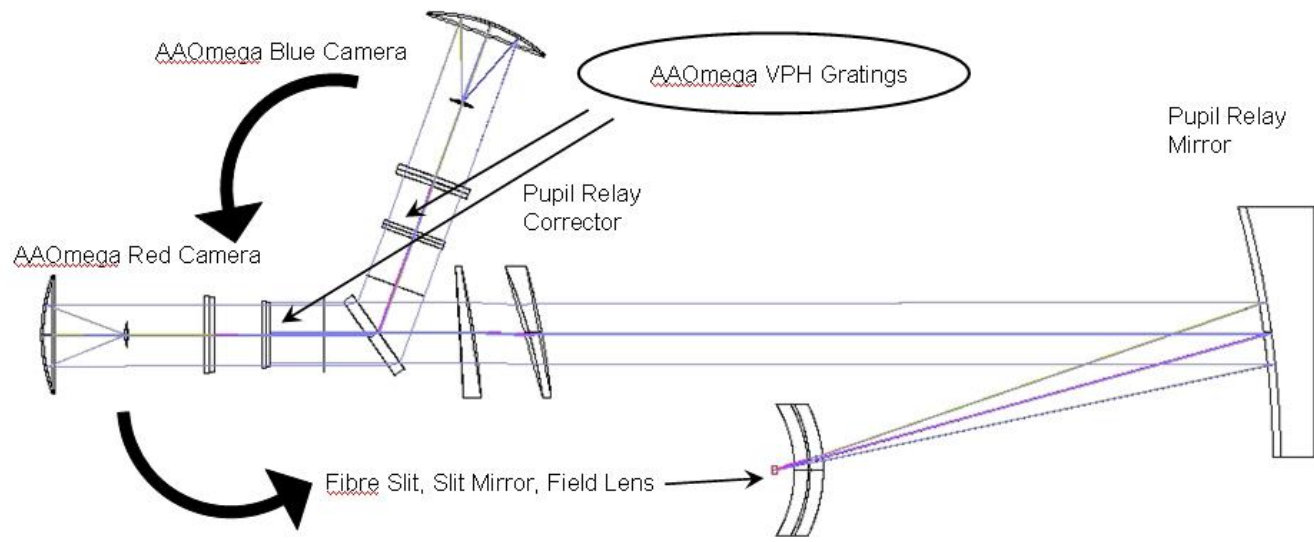

Echelle Grating moved out of way for camera articulation.

L

Fig 2. Optical schematic of the HERMES concept in the Low Resolution Mode. The fibre slit is moved and aligned to target the light towards the upper half of the collimator (the pupil relay mirror). The light then enters the AAOmega unit and is separated into two channels via the initial beam splitter. The transmissive cameras and related beam splitters are removed and replaced with the AAOmega VPH gratings. The final spectra are imaged onto the two Schmidt cameras. The echelle grating is removed in order to allow the full range of articulation angles required for classic AAOmega operation.

\section{INSTRUMENT PERFORMANCE}

\subsection{Wavelength Coverage}

In order to simultaneously sample the required number of spectral diagnostics needed for chemical tagging, a minimum spectral coverage of $400 \AA$ is required. At a resolving power of 30000 , this results in a coverage of 2000 resolution elements for a nominal wavelength of $6000 \AA$. With 3.8 pixels per resolution element, HERMES must have of order 7600 detector pixels in the spectral dimension. The two existing AAOmega cameras provide 4096 total spectral pixels or roughly half of that required. Two additional cameras are included in the HERMES High Resolution Mode in order to achieve the full spectral coverage with a total of 8192 pixels.

Figure 3 shows a plot of wavelength versus diffraction angle for the $110 \mathrm{l} / \mathrm{mm}$ R3 echelle. Vertical lines indicate the detector boundaries and a set of symbols indicate spectral indicators of interest for the stellar survey. Initial spectral orders of interest are shown in Table 4.

Table 4. Primary wavelength regions of interest for the stellar survey assuming that the dichroics can be produced with clean and efficient wavelength separation. Replacing order 28 with order 34 (5056 $\AA$ ) would provide more wavelength separation.

$\begin{array}{llllll}\text { Spectral Order: } \mathbf{m}\left(\boldsymbol{\lambda}_{\text {central }}\right) & 30(5730 \AA) & 28(6140 \AA) & 26(6612 \AA) & 20(8596 \AA) & \text { Total } \\ \text { Detector Wavelength Coverage } & 118 \AA & 127 \AA & 137 \AA & 178 \AA & 560 \AA\end{array}$




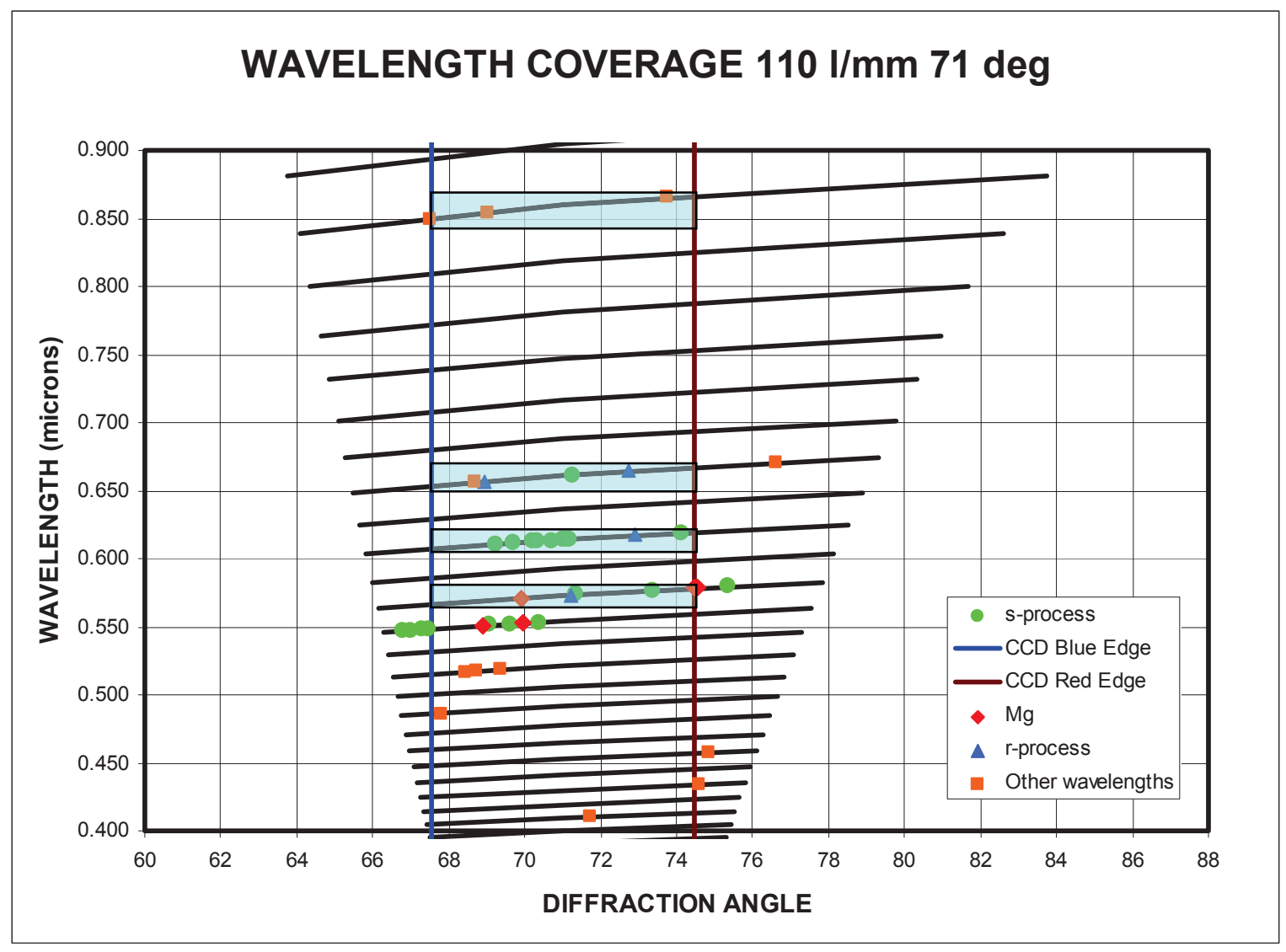

Fig 3. Wavelength as a function of diffraction angle for the $1101 / \mathrm{mm} \mathrm{R} 3$ echelle grating. The primary spectral orders of interest for the stellar survey are highlighted.

\subsection{Slit Curvature}

Nominal wavelength coverage is straightforward as described above. Unfortunately, there is a second order term in the diffraction equation for gratings that is related to the illumination angle with respect to the vector normal to the spatial plane of the grating. If that angle is non-zero, the diffraction angle is modified by a factor of $\cos (\gamma)$ where $\gamma$ is the incident angle in the plane parallel to the lines or grooves of the grating as related in the general grating equation ${ }^{[15]}$.

$$
m v \lambda=(\sin \alpha+\sin \beta) \cos \gamma
$$

where $m$ is the order of diffraction, $v$ is the line frequency, $\lambda$ is the wavelength, $\alpha$ is the angle of incidence in the plane perpendicular to the lines or grooves of the grating, and $\beta$ is the diffracted angle. For short slits this effect can usually be ignored as $\cos \gamma \sim 1$. However, in HERMES this effect becomes problematic due to the long slit length and high angles of incidence and diffraction. Figure 4 shows the effect of slit curvature on the detector for the R3 echelle for HERMES. As can be seen the effect is quite dramatic and causes the detector formats to be very inefficient at effective and uniform wavelength coverage for all observed targets.

Spectral coverage can be improved by curving the entrance slit to counter the slit curvature. Figures 5 and 6 show the effect of allowing a curved slit and the resulting detector coverage which is effectively uniform across all fibres.

Unfortunately a fixed curvature on the slit would mean that the Low Resolution Mode would have severe curvature in the opposite direction. To prevent this, a reconfigurable slit design is being explored in which the curvature can be changed between the major modes. Alternatively, a second fibre cable could be produced with a fixed slit curvature, but this likely adds additional costs as the fibre probes in $2 \mathrm{dF}$ would need to accommodate two fibres per probe.

The current optical design for HERMES includes a curved slit. 


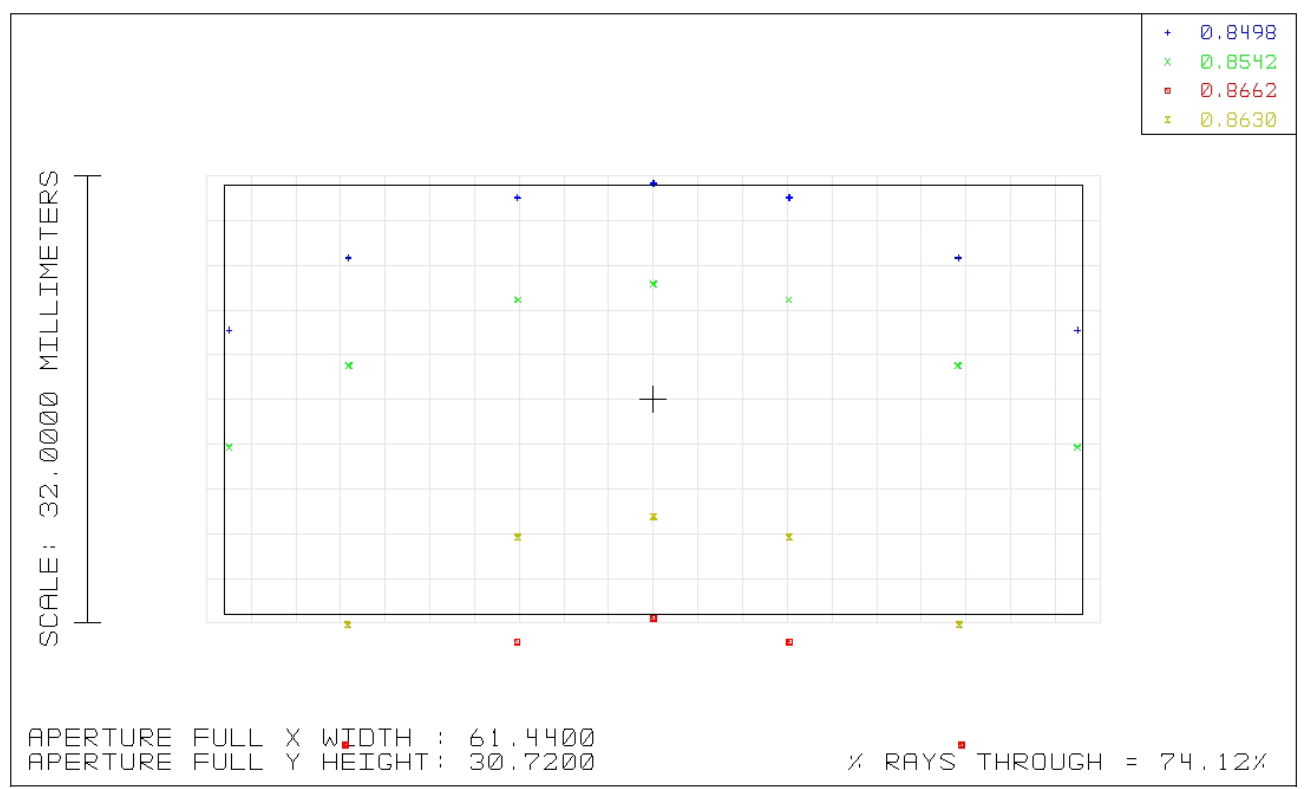

Fig 4. Detector coverage of the Ca II infrared triplet region with the R3 echelle and a straight slit.

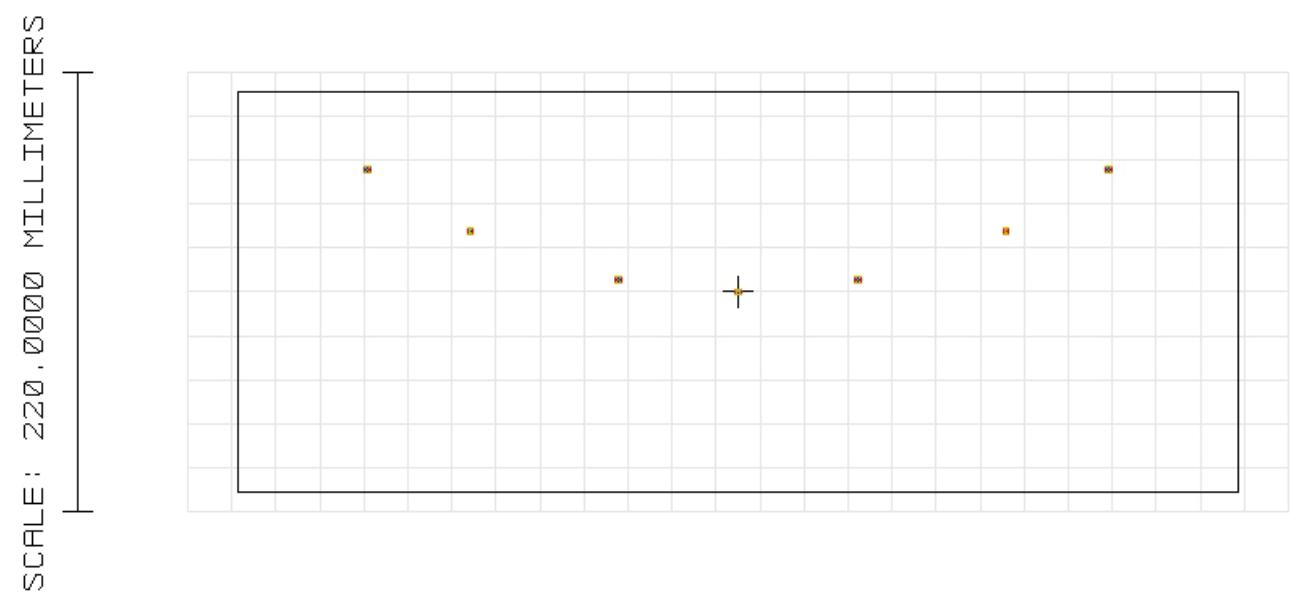

Fig 5. Slit curvature required to cancel the wavelength curvature shown in Figure 4.

\subsection{System Efficiency}

Figure 7 displays representative estimates for the High Resolution Mode of HERMES. Reasonable efficiency curves were applied to all components of the telescope, $2 \mathrm{dF}$ system, fibre cable, and spectrograph. The curves do not include the efficiency of the echelle grating, which is expected to peak at $65 \%$, nor do they include seeing losses on the fibre aperture. They do include estimates for the peak efficiency expected for the dichroics and bandpass filters, but do not include bandwidth limits that would be imposed by those filters.

\subsection{Optical Imagery}

Table 5 lists encircled radii at various positions within the image plane for the AAOmega Schmidt cameras in the High Resolution Mode. Image quality appears to be acceptable but may need improvement if a slit mask is to be used for the Very-High Resolution Mode. 


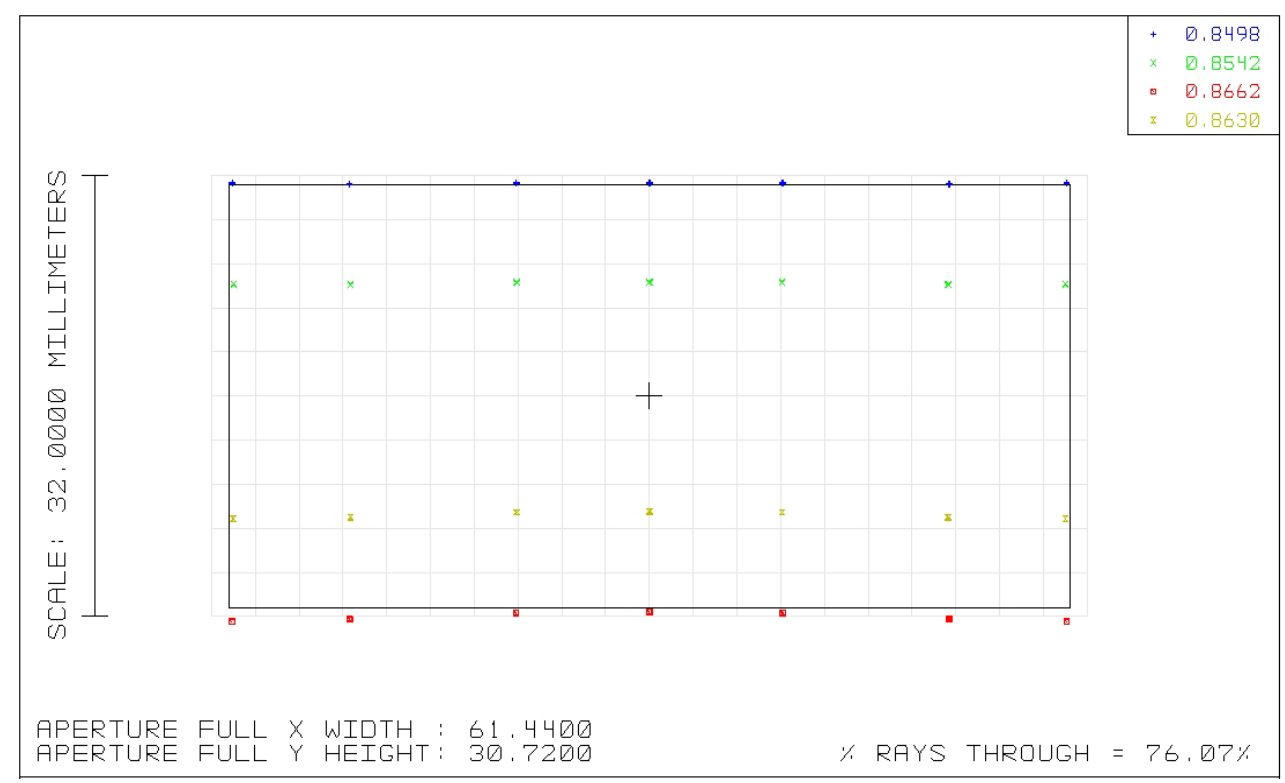

Fig 6. Resultant wavelength coverage on the detector if the slit is curved as shown in Figure 5.
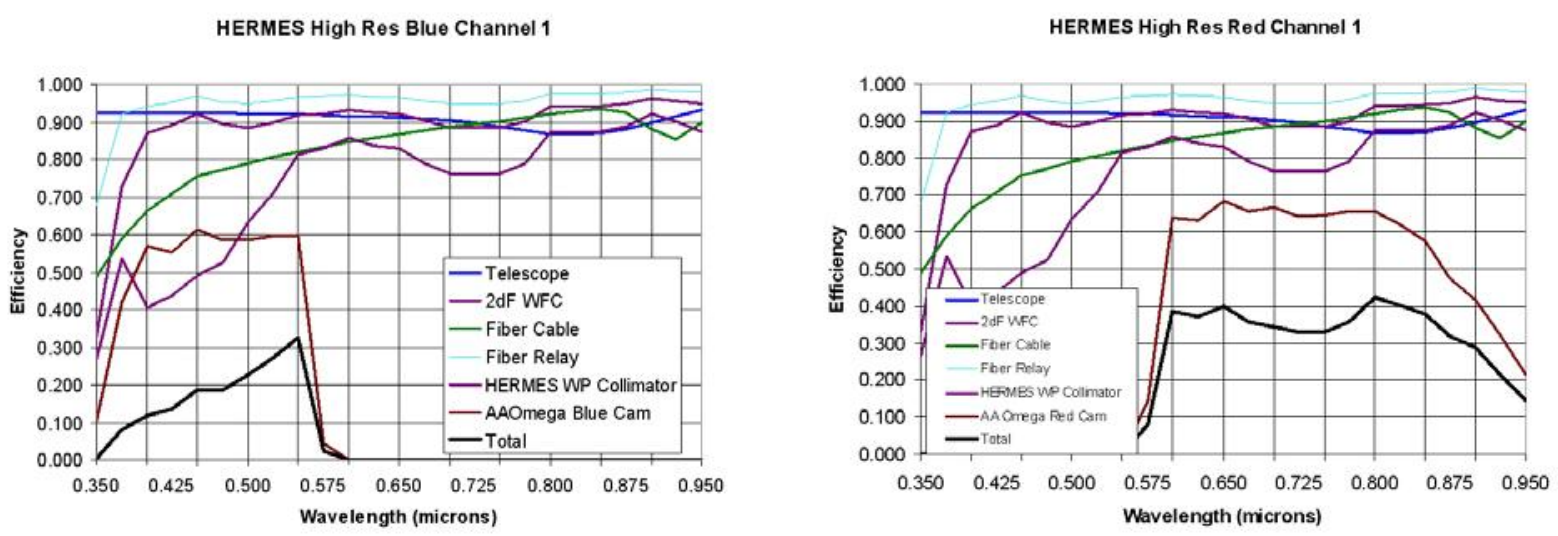

Fig 7. Efficiency models for HERMES. Left shows a model for the Blue AAOmega camera in the High Resolution Mode. The right panel shows the Red AAOmega camera for the same mode.

\section{CHALLENGES}

There are numerous technical challenges in the HERMES concept. Creating a reconfigurable curved slit is one such challenge, especially if the instrument is to be reconfigurable between the High and Low Resolution Modes. This is one of the arguments for examining the possible advantages of making HERMES a completely separate instrument from AAOmega. Fitting the instrument into the available space is another area of concern and one that appears to require modifications to the existing AAOmega lab. This space issue is another reason for looking at the option of HERMES and AAOmega remaining as separate entities. A view of how HERMES might fit into the existing space is shown in Figure 8. Reconfiguration of the downward looking cameras when switching between modes is another area of concern and one which would disappear if HERMES were a separate entity. The dichroic beam splitters are also likely to be technically challenging given their size (200 to $300 \mathrm{~mm}$ diameter) and the need for a relatively quick transition between the split wavelength regimes. Secondary spectral orders have been selected in case the primary regions can not be achieved. These are all design issues that will be examined in the coming year. 
Table 5. 80\% Encircled Energy Radii across the operating range of HERMES with the AAOmega cameras in High Resolution Mode.

\begin{tabular}{|ccccc|}
\hline $\begin{array}{c}\text { AOmega } \\
\text { Camera }\end{array}$ & $\begin{array}{c}\text { Wavelength } \\
(\mathrm{nm})\end{array}$ & $\begin{array}{c}\text { On-axis, } \\
80 \% \text { EER, } \\
\text { microns }\end{array}$ & $\begin{array}{c}0.7 \text { field } \\
80 \% \text { EER } \\
\text { microns }\end{array}$ & $\begin{array}{c}\text { Full-field } \\
80 \% \text { EER } \\
\text { microns }\end{array}$ \\
& 849.3 & 10.5 & 10 & 9 \\
& 857.3 & 18 & 14.5 & 12.5 \\
& 863.7 & 16.5 & 14 & 11 \\
& 566.2 & 8.5 & 13 & 10 \\
& 571.5 & 9 & 12.5 & 13 \\
& 575.8 & 10 & 11.5 & 10.5 \\
& 547.9 & 7.5 & 8 & 10 \\
& 553.05 & 16.5 & 10.5 & 7 \\
& 557.2 & 16.5 & 10 & 8.5 \\
& 369.2 & 16.5 & 19 & 16.5 \\
& 372.7 & 6 & 15 & 12.5 \\
& 375.5 & 4 & 6.5 & 15 \\
& & & & \\
& & 6 & &
\end{tabular}

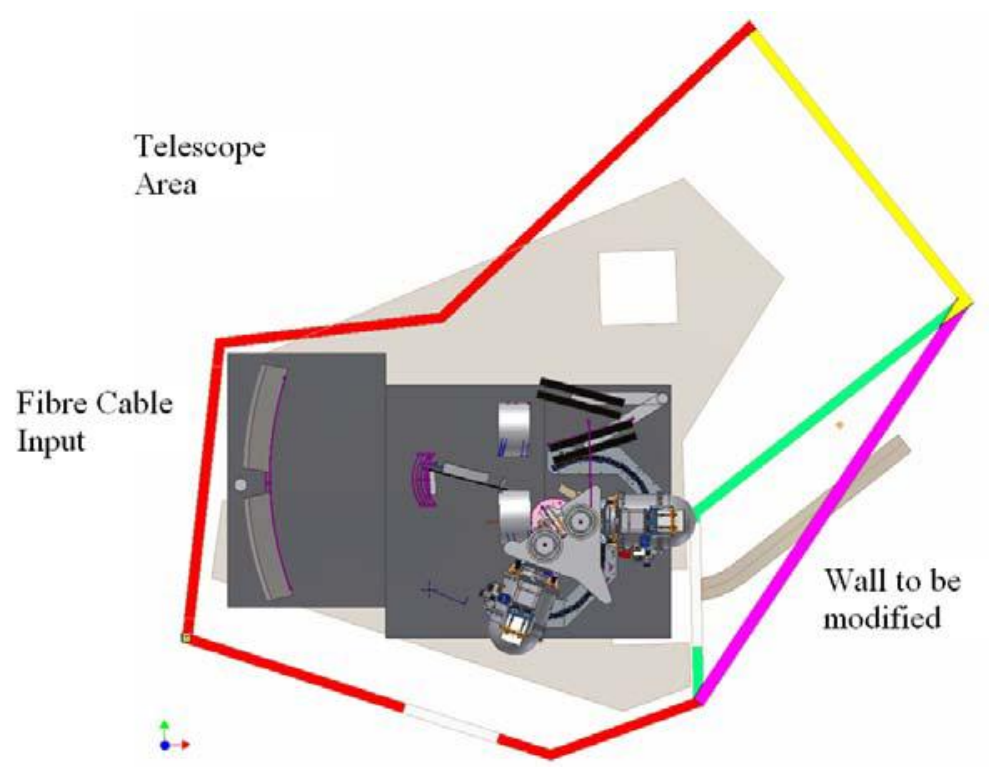

Fig 8. Layout of HERMES in the existing AAOmega lab indicating the wall that will require modification. 


\section{REFERENCES}

[1] Bland-Hawthorn, J. and Freeman, K. C., "Galactic history: formation \& evolution", Memorie della Societa Astronomica Italiana, 77, 1095-1102 (2006).

[2] Bland-Hawthorn, J. and Freeman, K. C., "Galaxy Genesis - Unravelling the Epoch of Dissipation in the Early Disk”, Pub. Astron. Soc. Australia, 21(2), 110-120 (2004).

[3] Freeman, K. and Bland-Hawthorn, J., "The New Galaxy: Signatures of Its Formation", Ann. Rev. Astron. and Astrophys., 40, 487-537 (2002).

[4] Sharp, R., Saunders, W., Smith, G., Churilov, V., Correll, D., Dawson, J., Farrell, T., Frost, G., Haynes, R., Heald, R., Lankshear, Al, Mayfield, D., Waller, L., and Whittard, D., "Performance of AAOmega: the AAT multi-purpose fibre-fed spectrograph", Proc. SPIE, 6269, 62690G-1-13 (2006).

[5] Smith, G. A., Saunders, W., Bridges, T., Churilov, V., Lankshear, A., Dawson, J., Correll, D., Waller, L., Haynes, R., and Frost, G., "AAOmega: a multipurpose fibre-fed spectrograph for the AAT", Proc. SPIE, 5492, 410-420 (2004).

[6] Saunders, W., Bridges, T., Gillingham, P., Haynes, R., Smith, G. A., Whittard, J. D., Churilov, V., Lankshear, A., Croom, S., Jones, D., and Boshuizen, C., “AAOmega: a scientific and optical overview”, Proc. SPIE, 5492, 389-400 (2004).

[7] Szentgyorgyi, A., "Hectochelle: High resolution multiobject spectroscopy at the MMT", New Astron. Rev., 50 (45), 326-328 (2006).

[8] Szentgyorgyi, A. H., Fabricant, D. G., Brown, W. R., and Epps, H. W., "Cross dispersion and an integral field unit for Hectochelle", Proc. SPIE, 4841, 1026-1035 (2003).

[9] Fabricant, D. G., Szentgyorgyi, A. H., and Epps, H. W., "Segmented Zero-Deviation Cross-Dispersion Prisms for the Hectochelle Multiobject Spectrograph", Pub. Astron. Soc. Pacific, 115 (804), 235-242 (2003).

[10] Cheimets, P., Szentgyorgyi, A. H., and Pieri, M. R., "Mosaicked echelle grating support for the Hectochelle fibrefed spectrograph", Proc. SPIE, 3355, 333-342 (1998).

[11] Szentgyorgyi, A. H., Cheimets, P., Eng, R., Fabricant, D. G., Geary, J. C., Hartmann, L., Pieri, M. R., and Roll, J. B., "Hectochelle: a multiobject echelle spectrograph for the converted MMT", Proc. SPIE, 3355, 242-252 (1998).

[12] Spano, P., Zerbi, F. M., Norrie, C. J., Cunningham, C. R., Strassmeier, K. G., Bianco, A., Blanche, P. A., Bougoin, M., Ghigo, M., Hartmann, P., Zago, L., Atad-Ettedgui, E., Delabre, B., Dekker, H., Melozzi, M., Snyders, B., and Takke, R., "Challenges in optics for Extremely Large Telescope instrumentation”, Astron. Nachr., 327 (7), 649-673 (2006).

[13] Baranne, A. and Duchesne, M., "Le spectrographe coudé "Echel.E.C.152" (Montage à pupille blanche pour caméra électronique)", Proc. ESO/CERN Conf. on Auxiliary Instrum. for Large Telescopes, 241-245 (1972).

[14] Baranne, A., "White Pupil Story or Evolution of a Spectrographic Mounting", Proc. ESO Conf. on Very Large Telescopes and their Instrumentation, ed. Ulrich, 1195 (1988).

[15] Loewen, E. G., and Popov, E., [Diffraction Gratings and Applications], Marcel Dekker, New York (1997). 